\title{
TWO DISCRETE FRACTIONAL INTEGRALS
}

\author{
DANiel M. Oberlin
}

The paper $[\mathrm{SW}]$ is a study of certain operators defined on the groups $\mathbb{Z}$ and $\mathbb{Z}^{2}$. These operators are analogues of fractional integrals along manifolds in $\mathbb{R}^{n}$, but, in the discrete setting, the manifolds are replaced by sets with certain arithmetic properties. In particular, for $0<\lambda<1$, Stein and Wainger introduce the operator $I_{\lambda}$, defined for functions $f$ on $\mathbb{Z}$ by

$$
I_{\lambda}(f)(n)=\sum_{m=1}^{\infty} \frac{f\left(n-m^{2}\right)}{m^{\lambda}}
$$

and the operator $J_{\lambda}$, defined for functions $f$ on $\mathbb{Z}^{2}$ by

$$
J_{\lambda}(f)\left(n_{1}, n_{2}\right)=\sum_{m=1}^{\infty} \frac{f\left(n_{1}-m, n_{2}-m^{2}\right)}{m^{\lambda}},
$$

and establish certain $\left(\ell^{p}, \ell^{q}\right)$ estimates for them. The approach in $[\mathrm{SW}]$ is to obtain these estimates by studying the multipliers associated with the operators. This is done by employing the "circle method" from number theory. The results obtained there continue a line of investigation dating back to Hardy and Littlewood and are extremely interesting in their own right. The purpose of this paper is to investigate the applicability to operators like $I_{\lambda}$ and $J_{\lambda}$ of two methods which have recently been used to study averaging operators associated with curves in $\mathbb{R}^{n}$. These methods make no use of the Fourier transform and so the "circle method" will not figure in our proofs. Nevertheless, and unsurprisingly, there are still connections with number theory.

One could abstract the ideas involved in the following proofs in order to fashion general theorems from which the results below would follow as special cases. Hoping to emphasize the simplicity of the current methods in this context, we have chosen not to do so.

We begin with the operator $I_{\lambda}$. Here the results of $[\mathrm{SW}]$ are the next theorem and corollary.

Received June 19, 2000. 
Theorem. Suppose $1 / 2<\lambda<1$. The operator $I_{\lambda}$ maps $\ell^{p}(\mathbb{Z})$ into $\ell^{q}(\mathbb{Z})$ when the following conditions hold:

$$
\begin{gathered}
1 / q \leq 1 / p-(1-\lambda) / 2 \\
p<1 /(1-\lambda), q>1 / \lambda .
\end{gathered}
$$

In case of equality in (i) there is the additional restriction that $p \leq 2 \leq q$. Conversely, if $0<\lambda<1$ and $I_{\lambda}$ maps $\ell^{p}(\mathbb{Z})$ into $\ell^{q}(\mathbb{Z})$, then (i) and (ii) must hold.

Corollary. Suppose $0<\lambda \leq 1 / 2$. The operator $I_{\lambda}$ maps $\ell^{p}(\mathbb{Z})$ into $\ell^{q}(\mathbb{Z})$ if the conditions $1 / q<1 / p-1+3 \lambda / 2, p<1 /(1-\lambda)$, and $q>1 / \lambda$ hold.

We will prove the following:

Theorem 1. Suppose $0<\lambda<1$ and $1 \leq p<q \leq \infty$. The operator $I_{\lambda}$ maps $\ell^{p}(\mathbb{Z})$ into $\ell^{q}(\mathbb{Z})$ if the conditions $1 / q<1 / p-(1-\lambda) / 2, p<1 /(1-\lambda)$, and $q>1 / \lambda$ hold.

Proof of Theorem 1. Interpolation arguments show that it is enough to check that $I_{\lambda}$ maps the Lorentz space $\ell^{\frac{3}{2}, 1}(\mathbb{Z})$ into $\ell^{3}(\mathbb{Z})$ whenever $\lambda=\frac{1}{3}+\epsilon$ and $\epsilon>0$. To this end, define

$$
I_{\lambda, j}(f)(n)=2^{-\lambda j} \sum_{m=2^{j}}^{2^{j+1}-1} f\left(n-m^{2}\right) .
$$

It is enough to show that

$$
\left\|I_{\lambda, j}\left(\chi_{F}\right)\right\|_{3} \leq C(\epsilon)|F|^{\frac{2}{3}}
$$

whenever $F \subseteq \mathbb{Z}$. Since

$$
\begin{aligned}
& \sum_{k \in \mathbb{Z}}\left(2^{-\lambda j} \sum_{m=2^{j}}^{2^{j+1}-1} \chi_{F}\left(k-m^{2}\right)\right)\left(2^{-\lambda j} \sum_{n=2^{j}}^{2^{j+1}-1} \chi_{F}\left(k-n^{2}\right)\right)^{2} \\
= & 2^{-3 \lambda j} \sum_{k \in \mathbb{Z}} \chi_{F}(k) \sum_{m=2^{j}}^{2^{j+1}-1}\left(\sum_{n=2^{j}}^{2^{j+1}-1} \chi_{F}\left(k+m^{2}-n^{2}\right)\right)^{2},
\end{aligned}
$$

(1) is equivalent to

$$
2^{-3 \lambda j} \sum_{k \in \mathbb{Z}} \chi_{F}(k) \sum_{m=2^{j}}^{2^{j+1}-1}\left(\sum_{n=2^{j}}^{2^{j+1}-1} \chi_{F}\left(k+m^{2}-n^{2}\right)\right)^{2} \leq C(\epsilon)|F|^{2}
$$


This inequality will be established using an argument from [O]:

setting $E=(F-k) \cap\left[-2^{2 j+2}, 2^{2 j+2}\right]$, it is enough to prove the inequality

$$
2^{-3 \lambda j} \sum_{m=2^{j}}^{2^{j+1}-1}\left(\sum_{n=2^{j}}^{2^{j+1}-1} \chi_{E}\left(m^{2}-n^{2}\right)\right)^{2} \leq C(\epsilon)|E|,
$$

for sets $E \subseteq\left[-2^{2 j+2}, 2^{2 j+2}\right]$. Thus, letting $S=S_{j}=\left\{n^{2}: 2^{j} \leq n<2^{j+1}\right\}$, we will show that

$$
\sum_{m=2^{j}}^{2^{j+1}-1}\left|E \cap\left(m^{2}-S\right)\right|^{2} \leq C(\epsilon) 2^{3 \lambda j}|E| .
$$

Since $|S|=2^{j}$, it follows that

$$
\sum_{m=2^{j}}^{2^{j+1}-1}\left|E \cap\left(m^{2}-S\right)\right|^{2} \leq 2^{j} \sum_{m=2^{j}}^{2^{j+1}-1}\left|E \cap\left(m^{2}-S\right)\right|=2^{j} \sum_{k \in E} \sum_{m=2^{j}}^{2^{j+1}-1} \chi_{\left(m^{2}-S\right)}(k),
$$

and so it is enough to check that

$$
\sum_{m=2^{j}}^{2^{j+1}-1} \chi_{\left(m^{2}-S\right)}(k) \leq C(\epsilon) 2^{3 \epsilon j}
$$

if $k \in E$. But if $\chi_{\left(m^{2}-S\right)}(k)=1$, there is $n$ such that $(m-n)(m+n)=k$. Then $n=\sqrt{m^{2}-k}$ so that $m+\sqrt{m^{2}-k}$ is a divisor of $|k|$. Since $m+\sqrt{m^{2}-k}$ is an increasing function of $m$, the number of such $m$ is bounded by $d(|k|)$, the number of divisors of $|k|$. Now $d(|k|)$ is $O\left(|k|^{\delta}\right)$ for any $\delta>0$ ([HW], p.260) and so (2) follows from $|k| \leq 2^{2 j+2}$ if $k \in E$. This completes the proof of Theorem 1.

The situation is somewhat analogous for the operator $J_{\lambda}$ on $\mathbb{Z}^{2}$. Here the results from $[\mathrm{SW}]$ are the following theorem and corollary.

Theorem. Suppose $1 / 2<\lambda<1$. The operator $J_{\lambda}$ maps $\ell^{p}\left(\mathbb{Z}^{2}\right)$ into $\ell^{q}\left(\mathbb{Z}^{2}\right)$ when the following conditions hold:

$$
\begin{aligned}
& 1 / q \leq 1 / p-(1-\lambda) / 3 \\
& p<1 /(1-\lambda), q>1 / \lambda .
\end{aligned}
$$

In case of equality in (i) there is the additional restriction that $p \leq 2 \leq q$.

Corollary. Suppose $0<\lambda \leq 1 / 2$. The operator $J_{\lambda}$ maps $\ell^{p}\left(\mathbb{Z}^{2}\right)$ into $\ell^{q}\left(\mathbb{Z}^{2}\right)$ if the conditions $1 / q<1 / p-1-5 \lambda / 3, p<1 /(1-\lambda)$, and $q>1 / \lambda$ hold.

Analogous to Theorem 1, we will establish 
Theorem 2. Suppose $0<\lambda<1$ and $1 \leq p<q \leq \infty$. The operator $J_{\lambda}$ maps $\ell^{p}\left(\mathbb{Z}^{2}\right)$ into $\ell^{q}\left(\mathbb{Z}^{2}\right)$ if the conditions $1 / q<1 / p-(1-\lambda) / 3, p<1 /(1-\lambda)$, and $q>1 / \lambda$ hold.

Proof of Theorem 2. Interpolation arguments show that it is enough to check that $J_{\lambda}$ is of restricted weak type $\left(\frac{5}{3}, \frac{5}{2}\right)$ whenever $\lambda=\frac{2}{5}+\epsilon$ and $\epsilon>0$. We begin by defining

$$
J_{\lambda, j}(f)\left(n_{1}, n_{2}\right)=2^{-\lambda j} \sum_{m=2^{j}}^{2^{j+1}-1} f\left(n_{1}-m, n_{2}-m^{2}\right) .
$$

Fixing $\epsilon>0$ and $\lambda=\frac{2}{5}+\epsilon$, it will suffice to show that the operators $J_{\lambda, j}$ are of restricted weak type $\left(\frac{5}{3}, \frac{5}{2}\right)$ uniformly in $j$. We will adapt some ideas from [C] and begin with a lemma, the proof of which is contained in the proof of Lemma 1 in $[\mathrm{C}]$.

Lemma. For $k=0,1, \ldots$ there are positive constants $\delta_{k}$ and $\epsilon_{k}$ such that the following holds. Suppose $T$ is a positive operator taking measurable functions $\chi_{E}$ on some measure space onto measurable functions. Suppose $\alpha>0, F=$ $\left\{\alpha<T \chi_{E}<2 \alpha\right\},|F|>0$, and $\beta=|E|^{-1}\left\langle\chi_{F}, T \chi_{E}\right\rangle$. Define $E_{0}=E, F_{0}=F$, $E_{k+1}=\left\{x \in E_{k}: T^{*} \chi_{F_{k}}(x) \geq \delta_{k} \beta\right\}$ and $F_{k+1}=\left\{y \in F_{k}: T \chi_{E_{k+1}}(y) \geq \epsilon_{k} \alpha\right\}$. Then all of the sets $E_{k}$ and $F_{k}$ are nonempty.

Fix $E \subseteq \mathbb{Z}^{2}$ and $\alpha>0$. Writing $T$ for $J_{\lambda, j}$ and $F=\left\{\alpha<T \chi_{E}<2 \alpha\right\}$ as above, the restricted weak type result we seek will follow from the estimate

$$
\alpha^{5}|F|^{2} \leq C(\epsilon)|E|^{3} .
$$

Let $x$ and $y$ stand for elements of $\mathbb{Z}^{2}$. Continuing to argue as in $[\mathrm{C}]$, we note that $x \in E_{k}$ implies

$$
\sum_{2^{j} \leq m<2^{j+1}} \chi_{F_{k-1}}\left(x+\left(m, m^{2}\right)\right) \geq 2^{\lambda j} \delta_{k-1} \beta
$$

and $y \in F_{k}$ implies

$$
\sum_{2^{j} \leq m<2^{j+1}} \chi_{E_{k}}\left(y-\left(m, m^{2}\right)\right) \geq 2^{\lambda j} \epsilon_{k-1} \alpha
$$

Fix $y \in F_{2}$ and consider the sum $S$ given by

$$
\sum_{2^{j} \leq m_{1}, \ldots, m_{5}<2^{j+1}} \chi_{E}\left(y-\left(m_{1}, m_{1}^{2}\right)+\left(m_{2}, m_{2}^{2}\right)-\left(m_{3}, m_{3}^{2}\right)+\left(m_{4}, m_{4}^{2}\right)-\left(m_{5}, m_{5}^{2}\right)\right) .
$$

Now (5) shows that there are at least $2^{\lambda j} \epsilon_{1} \alpha$ values of $m_{1}$ with $y-\left(m_{1}, m_{1}^{2}\right) \in E_{2}$. For each of these, (4) shows that there are at least $2^{\lambda j} \delta_{1} \beta$ values of $m_{2}$ with 
$y-\left(m_{1}, m_{1}^{2}\right)+\left(m_{2}, m_{2}^{2}\right) \in F_{1}$. Continuing this process, and recalling that $E_{0}=E$, leads to

$$
S \geq 2^{5 \lambda j} \epsilon_{0} \delta_{0} \epsilon_{1} \delta_{1} \alpha^{3} \beta^{2} \geq c 2^{5 \lambda j} \alpha^{5}\left(\frac{|F|}{|E|}\right)^{2}
$$

where the second inequality follows from $\beta \geq \alpha|F| /|E|$. Let $\delta=5 \epsilon$ so that $5 \lambda=2+\delta$. Then for some fixed $m_{2}, m_{4}$ we have

$\sum_{2^{j} \leq m_{1}, m_{3}, m_{5}<2^{j+1}} \chi_{E}\left(y-\left(m_{1}, m_{1}^{2}\right)+\left(m_{2}, m_{2}^{2}\right)-\left(m_{3}, m_{3}^{2}\right)+\left(m_{4}, m_{4}^{2}\right)-\left(m_{5}, m_{5}^{2}\right)\right)$

$$
\geq \frac{S}{2^{2 j}} \geq c 2^{\delta j} \alpha^{5}\left(\frac{|F|}{|E|}\right)^{2}
$$

Thus (3) will follow from the estimate

$$
\begin{gathered}
\sum_{2^{j} \leq m_{1}, m_{3}, m_{5}<2^{j+1}} \chi_{E}\left(y-\left(m_{1}, m_{1}^{2}\right)+\left(m_{2}, m_{2}^{2}\right)-\left(m_{3}, m_{3}^{2}\right)+\left(m_{4}, m_{4}^{2}\right)-\left(m_{5}, m_{5}^{2}\right)\right) \\
\leq C(\delta)|E| 2^{\delta j} .
\end{gathered}
$$

Replacing $E$ by $y+\left(m_{2}, m_{2}^{2}\right)+\left(m_{4}, m_{4}^{2}\right)-E$, we have

$$
\begin{aligned}
\sum_{2^{j} \leq m_{1}, m_{3}, m_{5}<2^{j+1}} \chi_{E}\left(\left(m_{1}, m_{1}^{2}\right)+\left(m_{3}, m_{3}^{2}\right)+\left(m_{5}, m_{5}^{2}\right)\right) & =\sum_{(a, b) \in E} \sum_{\substack{m_{1}+m_{3}+m_{5}=a \\
m_{1}^{2}+m_{3}^{2}+m_{5}^{2}=b}} 1
\end{aligned}
$$

where, in the last sum, $2^{j} \leq m_{1}, m_{3}, m_{5}<2^{j+1}$. It is therefore enough to observe the following lemma.

Lemma. For $(a, b) \in \mathbb{Z}^{2}$ the number of solutions in positive integers $m_{j}$ to the simultaneous equations $m_{1}+m_{2}+m_{3}=a$ and $m_{1}^{2}+m_{2}^{2}+m_{3}^{2}=b$ is $O\left(\left(b+a^{2}\right)^{\delta}\right)$ for any $\delta>0$.

Proof of Lemma. Setting $n_{j}=3 m_{j}-a$ leads to the equations $n_{1}+n_{2}+n_{3}=0$, $\left(n_{1}+a\right)^{2}+\left(n_{2}+a\right)^{2}+\left(n_{3}+a\right)^{2}=9 b$ and hence to $n_{1}^{2}+n_{1} n_{2}+n_{2}^{2}=\left(9 b-3 a^{2}\right) / 2$. The equation $n_{1}^{2}+n_{1} n_{2}+n_{2}^{2}=n$ is solvable in integers only if $n=3^{\alpha} \Pi p^{\beta} \Pi q^{\gamma}$ where the primes $p$ are of the form $3 k+1$, the primes $q$ are of the form $3 k+2$, and all the exponents $\gamma$ are even. Then the number of solutions is $\Pi_{p}(\beta+1)$. (See problem 4 on page 176 of $[\mathrm{NZM}]$, for example.) It is easy to check that $\Pi_{p}(\beta+1)$ is $O\left(\left(\Pi p^{\beta}\right)^{\delta}\right)$ for any $\delta>0$. This gives the lemma and concludes the proof of Theorem 2 .

The analogue of Theorem 1 with $m^{2}$ in the definition of $I_{\lambda}$ replaced by $m^{k}$ would require replacing the inequality $1 / q<1 / p-(1-\lambda) / 2$ in the statement of 
Theorem 1 by $1 / q<1 / p-(1-\lambda) / k$. An analogy between the powers $m^{k}$ in $\mathbb{Z}$ and the curve $\left(t, t^{2}, \ldots, t^{k}\right)$ in $\mathbb{R}^{k}$ tempts one to try to apply the ideas from $[\mathrm{C}]$ to prove the (conjectured) result for $m^{k}$. Things go smoothly until the end, where one requires the following number-theoretic result: the number of representations of a nonnegative integer $n$ as a sum of $k k^{t h}$ powers of nonnegative integers is $O\left(n^{\delta}\right)$ for any $\delta>0$. This is true when $k=2$ (and thus the method of proof of Theorem 2 could be adapted to yield Theorem 1 as well) but is stronger than the "Hypothesis $\mathrm{K}^{*}$ " (see the discussion in $[\mathrm{SW}]$ ) and is therefore an open question for $k>2$.

Note. Shortly after this paper was completed, Stein and Wainger (oral communication) were able to strengthen the conclusions of Theorems 1 and 2. Their results replace the inequality $1 / q<1 / p-(1-\lambda) / 2$ of Theorem 1 by the equality $1 / q=1 / p-(1-\lambda) / 2$ and the inequality $1 / q<1 / p-(1-\lambda) / 3$ of Theorem 2 by the equality $1 / q=1 / p-(1-\lambda) / 3$. These were obtained by a compli-

cated argument involving the circle method and certain decompositions of the operators.

\section{References}

[C] M. Christ, Convolution, curvature, and combinatorics, a case study, Internat. Math. Res. Notices 19 (1998), 1033-1048.

[O] D. Oberlin, Convolution with affine arclength measures in the plane, Proc. Amer. Math. Soc. 127 (1999), 3591-3592..

[HW] G.H. Hardy and E. M. Wright, An introduction to the theory of numbers, 3rd edition, Clarendon Press, Oxford, 1954..

[NZM] I. Niven, H. S. Zuckerman, and H. L. Montgomery, An introduction to the theory of numbers, 5th edition, John Wiley \& Sons Inc., New York, 1991.

[SW] E. M. Stein and S. Wainger, Discrete analogues in harmonic analysis II: fractional integration, J. Anal. Math. 80 (2000), 335-355.

Dept. of Mathematics, Florida State University, Tallahassee, FL 32306-4510.

E-mail address: oberlin@math.fsu.edu 